\title{
Discovering and Implementing Self-Sustaining Networks of Cooperation with General Collective Intelligence
}

Andy E. Williams

\begin{abstract}
Leveraging General Collective Intelligence or GCI, a platform with the potential to achieve an exponential increase in general problem-solving ability, a methodology is defined for finding potential opportunities for cooperation, as well as for negotiating and launching cooperation. This paper explores the mechanisms by which GCI enables networks of cooperation to be formed in order to increase outcomes of cooperation and in order to make that cooperation self-sustaining. And this paper explores why implementing a GCI for the first time requires designing an iterative process that self-assembles continually growing networks of cooperation.
\end{abstract}

\section{Keywords}

General Collective Intelligence, networks of cooperation, chains of cooperation

\section{Introduction}

General Collective Intelligence or GCI [1] is a platform with the potential to achieve an exponential increase in general problem-solving ability (intelligence) over that of any individual. One way this is achieved is through defining common functional models of reasoning processes that can be shared, and increasing the complexity of problems that can be defined or solved by potentially incorporating all the information and reasoning processes possessed by every individual in the group, increasing capacity for cooperation by potentially incorporating all individuals and all their computing devices and other resources, and increasing capacity to sustain these efforts by engaging all individuals in processes that create sufficient value to be self-sustaining.

In order to scale outcomes of problem-solving or any other process in this way by scaling the processes that can be executed, by scaling the number of entities involved in executing those processes as well as the resources engaged by those entities, and by combining processes into networks of cooperation that create enough value for the cooperation to be self-sustaining, all processes must be decoupled from any individual, any resource, or any other attribute of processing so that they might be executed elsewhere. In the case of processes automated by software applications, decoupling attributes such as physical device, identity, or security from the actual application process being executed might be achieved through a platform such as the proposed Peer to Peer Social Fabric (P2PSF) [2], so that the process can be scaled in whatever way that is required to increase outcomes.

Functional models can be defined for virtually any group process, whether involving physical activity, or virtual. By defining functional models of group processes as consisting of activities that can be decoupled by a GCI platform, those activities can be executed in any complex combination to achieve different outcomes, those activities can be executed in series or in parallel by additional individuals in each role to scale those outcomes, and outcomes can be optimized across all processes of cooperation to create enough value through that cooperation to make it self-sustaining.

\section{Chains of Cooperation}

Simple cooperation between two parties can be represented by a first order barter transaction in which one entity exchanges some value in return for the other party exchanging an approximately equal value. Complex cooperation can be represented by Nth order barter transactions in which the first party gives something to the second party, who gives something to the third party, and so on until the $\mathrm{N}-1$ th party 
gives something to the Nth party. Barter transactions can be open in the sense that someone outside of the network provides the demand that supports the entire chain of cooperation, or they can be closed.

\section{Nth Order Network Barter Transactions}

\section{Open Network}

Barter

Transaction

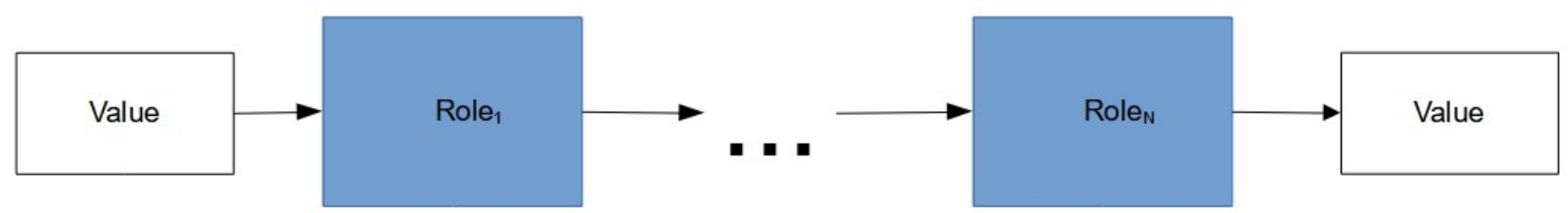

\section{Closed Network}

\section{Barter}

Transaction

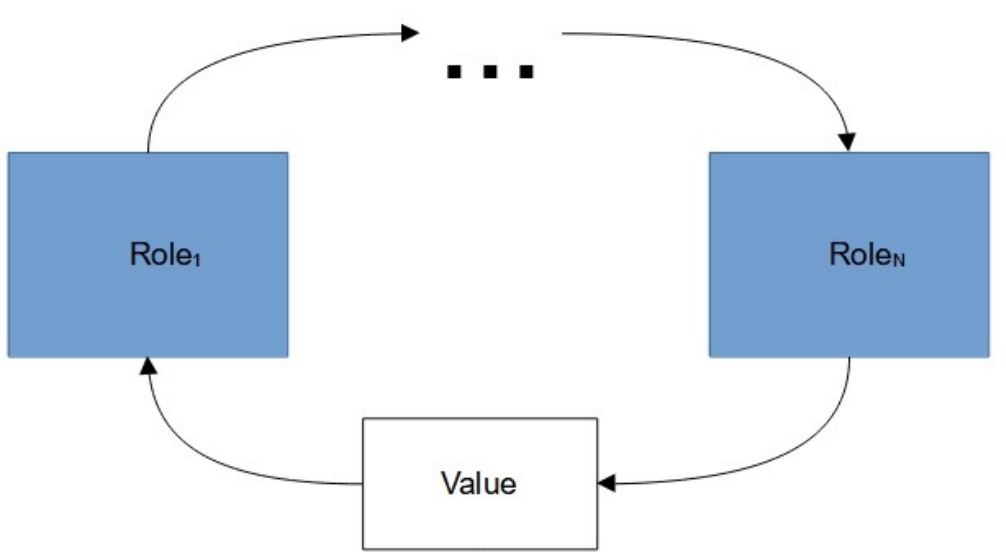

Figure 1: Network barter transactions might be open or closed.

And they can also be hierarchical in that all participants exchange value with a single entity and in return share the value provided by that entity. 


\section{Nth Order Hierarchical Barter Transactions}

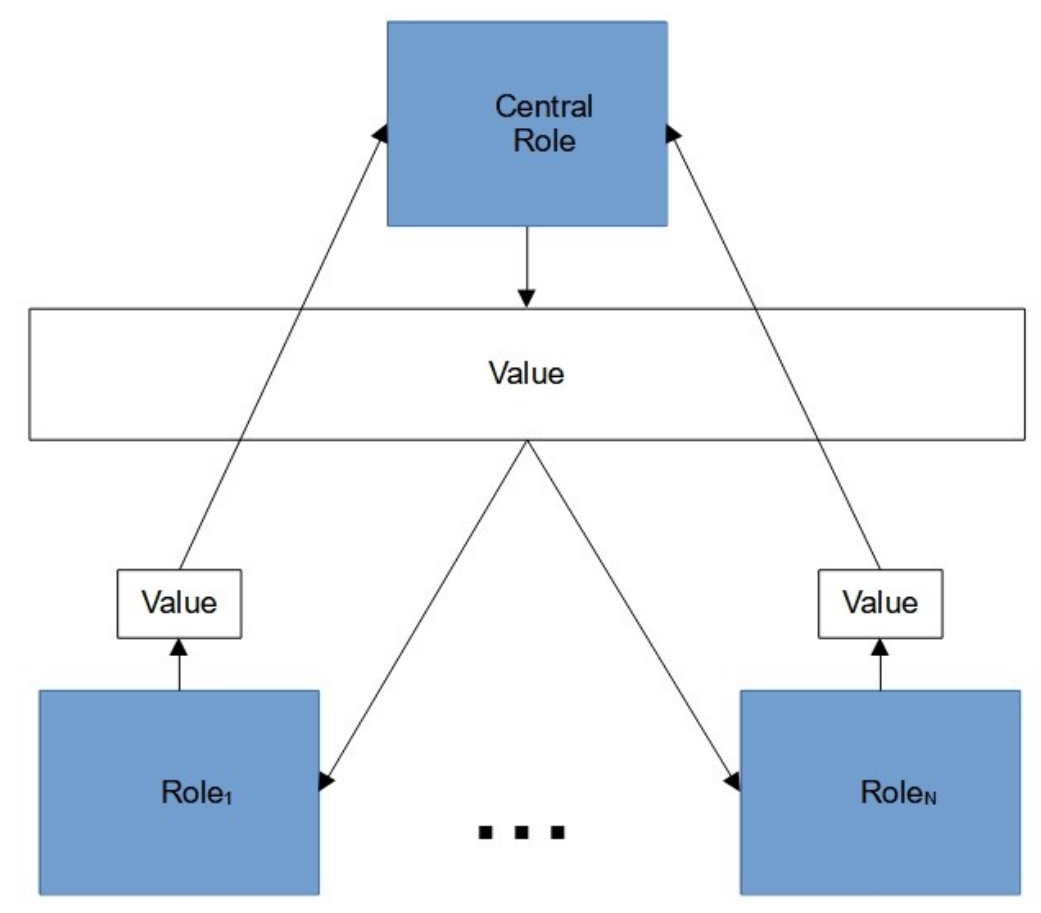

Figure 2: Hierarchical barter transactions involve a central role.

An example would be a group of farmers who each give a proportion of their crop to a road builder who in return builds a road to market they all can use. Nth order barter transactions can enable a GCI to optimize collective outcomes over an unlimited number of possible chains of cooperation, to the capacity of the cooperating entities to engage in that cooperation.

However, a single open chain of cooperation might continually require inputs from outside the chain of cooperation, and might continually produce outputs outside the chain of cooperation. Those inputs or outputs might not be sustainable. 


\section{Flow of Value Requiring Continual External Input}

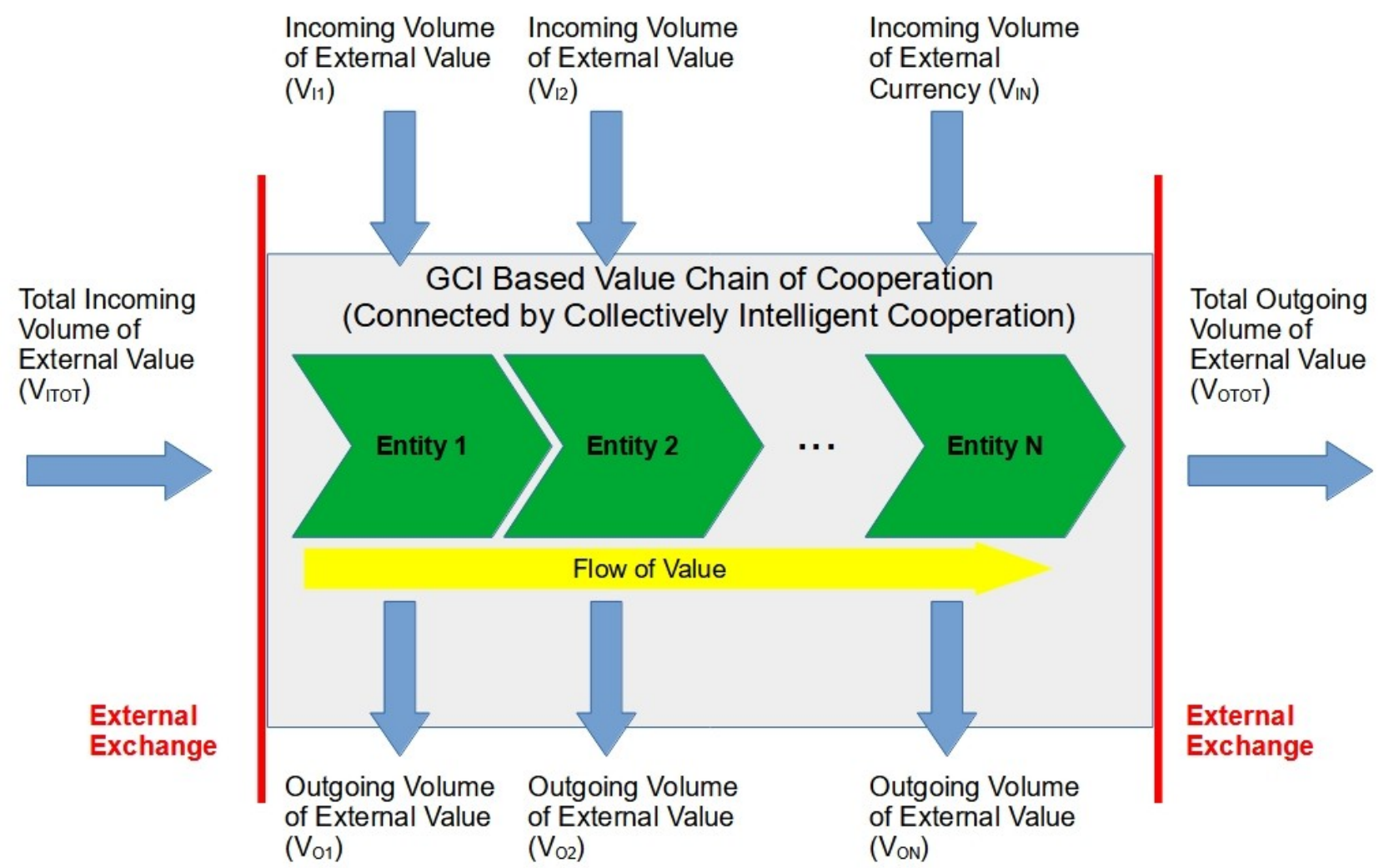

Figure 3: The value required from outside the chain of cooperation might make the chain of cooperation unsustainable.

In order for a chain of cooperation to be feasible, the cooperation must create some total value so that part of that value can be used to incentivize the participation of each entity. If the value of the cooperation can be objectively projected, and if the cooperation is rewarded with some fraction of the value actually achieved, then cooperation is always potentially feasible. Of course, any entity can only engage in so many opportunities at one time.

If opportunities for feasible cooperation can be assessed and engaged in an automated way up to each entity's capacity, it might be possible to realize the value of this cooperation at much higher speed and scale. In the case of open chains of cooperation for which the demand required to support the entire chain of cooperation comes from outside it, Feasibility of that cooperation depends on the ability to ensure that demand. Ensuring this demand might be achieved by unevenly distributing the value from cooperation so that the value in the cooperation can be used to create whatever incentive is sufficient to create that demand. For example, consider the case of a country in which all school uniforms are imported from outside the country, all cotton grown inside the country is exported, and as a result of the lack of any clothes making industry there are a lack of jobs. A value chain based on collectively intelligent cooperation might address this. In collectively intelligent cooperation one business agrees to buy some key product or service from another business in the chain in return for another business agreeing to buy from it. Such a value chain might involve a distributor to sell locally made school uniforms, a manufacturer of those uniforms, a company to train tailors for that manufacturing, a local textile weaver, and a local source of cotton. In this collectively intelligent cooperation, in return for a commitment from customers the distributor agrees to buy uniforms from the manufacturer, in return for 
a commitment from the distributor the manufacture agrees to buy cloth from the textile factory and to hire tailors, in return for a commitment from the manufacturer the textile factory agrees to buy cotton from the source and the training company agrees to train tailors. By each participant agreeing to buy a key product or service from another business in the value chain in return for another business agreeing to buy from them, then even before they start they have forward contracts that might provide enough security to ensure they can access the finance and everything else they need to launch. In order to ensure demand the distributor might give all stores that sell school uniforms a $50 \%$ discount for the first year, or whatever discount required to undercut imported uniforms, in return for those stores signing up to procure school uniforms from them for the next five years, with the discount being paid out over time to ensure compliance. In order to pay for this discount the distributor, trainer, manufacturer, textile weaver, and cotton source might each contribute $10 \%$ to this subsidy. While the subsidy required to reliably ensure demand might be too large for the distributor to give alone, with collectively intelligent cooperation that demand supports an entire value chain so it can cooperate to give that subsidy. As the side of the value chain increases, the possible subsidy it can potentially provide grows to the point that it might provide unbeatable competitive advantage.

\section{Networks of Cooperation}

In addition to using cooperation to ensure demand so that a single chain of cooperation is sustainable, chains of cooperation can also be combined into networks designed to exchange value between the chains in a way such that the flow of value in the entire network is sustainable.

\section{Self-Sustaining Flow of Value}

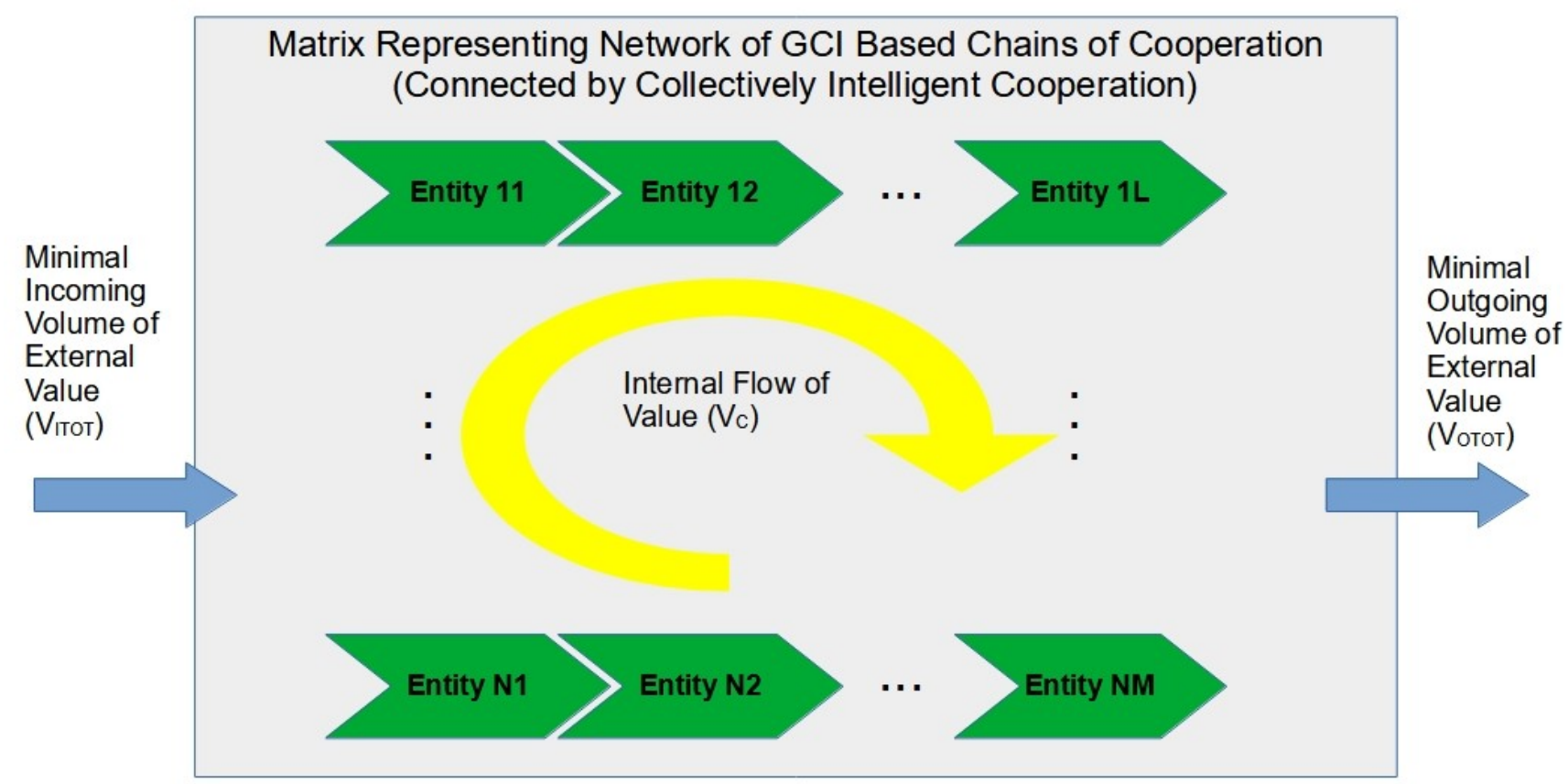

Figure 4: The value created within a network of cooperation can make all the chains of cooperation sustainable.

\section{Deploying Networks of Cooperation}


The creation and deployment of a General Collective Intelligence is a complex process that is itself believed to require GCI to be reliably achievable. To get over this chicken and egg self-dependency, an iterative evolutionary approach might be used. In this approach an individual or team might build the small subset of GCI functionality that they can achieve on their own, and then use that functionality to engage more people to build more GCI functionality, which might ten used to engage more people and build still more GCI functionality, in an iterative cycle.

GCI based collectively intelligent cooperation of course requires GCI. Because GCI has not yet been implemented, this cooperation, or any other GCI based process, must iteratively build a subset of GCI functionality, and then use that functionality to create value in order to engage a larger group to participate in leveraging that value, then using that GCI functionality to build more GCI functionality that creates yet more value, and so forth in an iterative cycle of engagement. For this to be achievable, GCI functionality must be decomposed into sets of functionality that can be implemented in each cycle, where these sets form a hierarchy in order of their dependencies. Therefore implementation of the first GCI based process must first implement the minimal subset of GCI that can be implemented by an individual or team without GCI. Assume some process has been defined to achieve an outcome with that minimal subset of GCI functionality.

The Collective Intelligence based Program to Accelerate Achievement of the Sustainable Development Goals (CIPAA-SDGs) is an example of a hierarchy of a great many projects designed to implement a GCI in an iterative cycle while using that increasing GCI functionality to multiply social impact. 


\section{Deployment of the Collective Intelligence based Program to Accelerate Achievement of the Sustainable Development Goals}

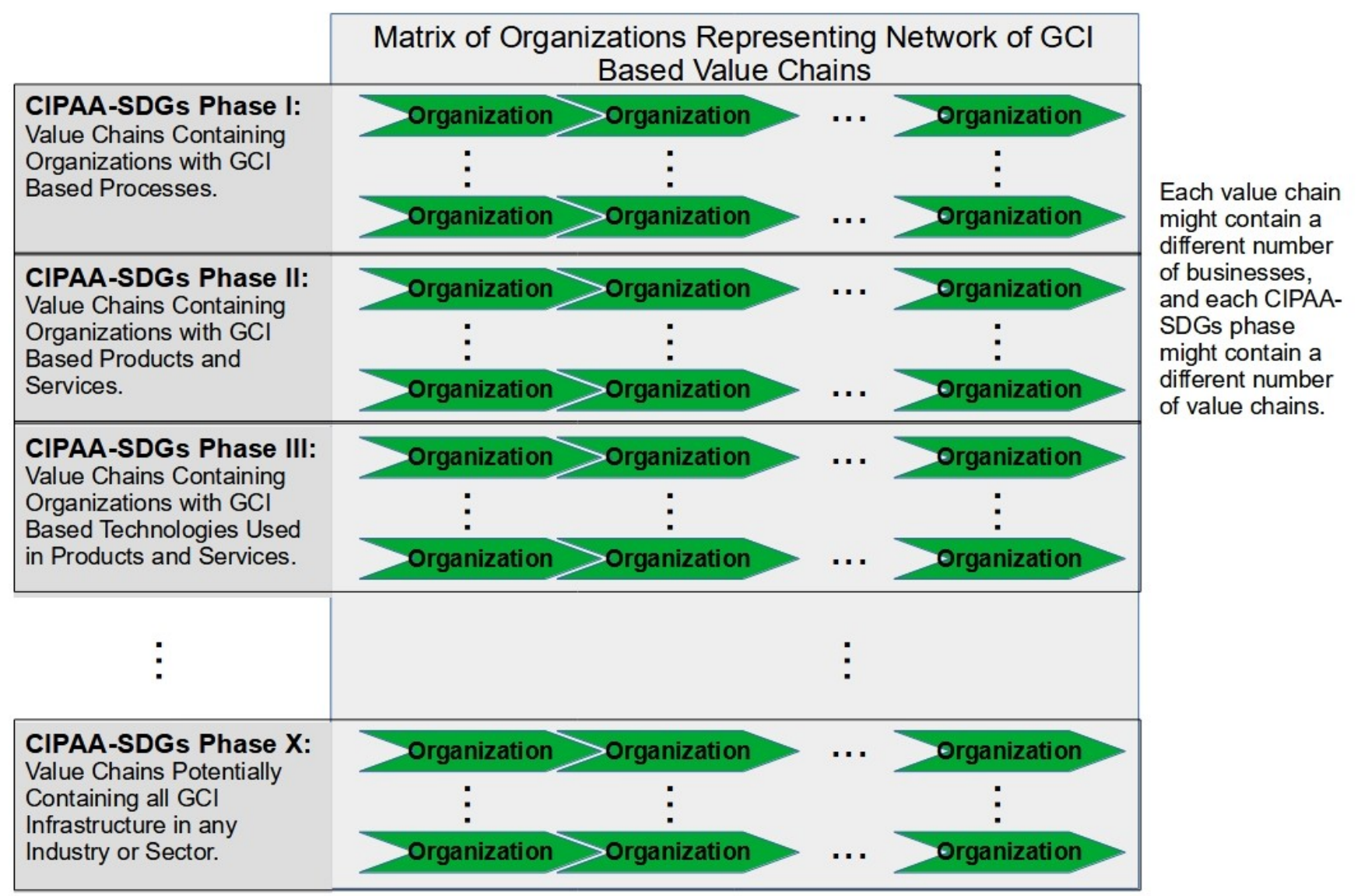

Figure 5: GCI must be deployed in a hierarchy of programs. The CIPAA-SDGs program defines such a hierarchy.

In general, deployment of any program involving a GCI based network of cooperation must be executed in such a hierarchy where that program is also used to implement GCI. 


\section{Deployment of a General Collective Intelligence Based Network of Cooperation}

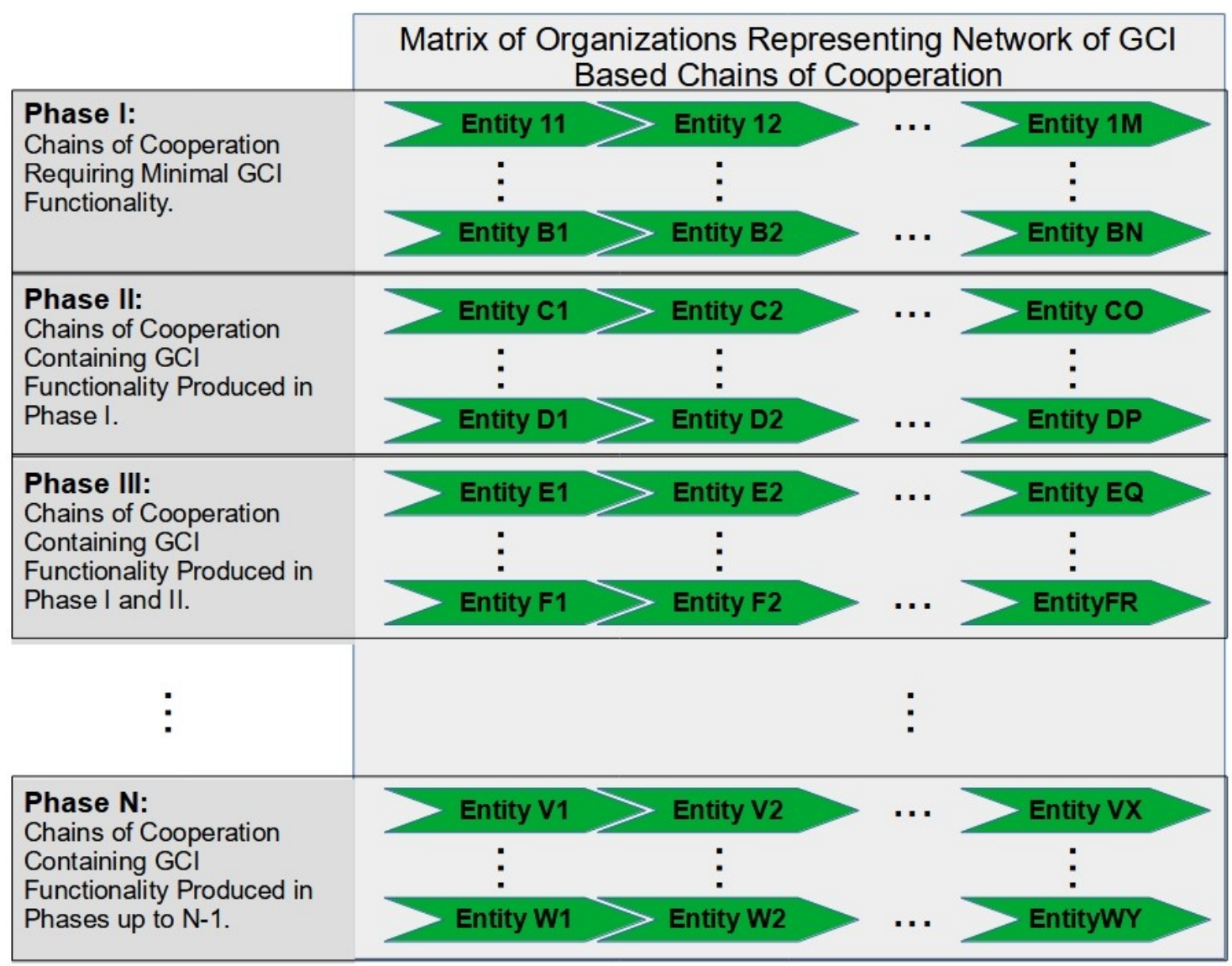

Figure 6: This pattern can be generalized to define other hierarchies of programs that might be used to deploy a GCI.

\section{Engaging the Effort Required to Deploy Networks of Cooperation}

Engaging the potentially great number of entities involved in such a network of cooperation requires understanding all factors that might limit capacity to engage.

On teams of limited size, a specific individual might be tasked with a given role. However in general, ensuring a specific individual has the required attributes to play a particular role in a process, and ensuring that they are incentivized do so, and that they have been engaged to do so, is not assured to be reliably achievable. The more difficult the task, and therefore the smaller the percentage of the population that can execute it, and the more specific the motivation, and therefore the smaller the percentage of the population that can be motivated to do so, the more unlikely it is to engage any individual in performing that task. As the type of incentive required to engage a specific individual to perform a specific role becomes more unobtainable, and as the value of that incentive increases with difficulty of the task and other factors, engagement of that specific individual might becomes less and less probable to the point that their engagement cannot be reliably achieved. Changing the process so that it just requires any individual who is most qualified to play that role, increasing the size of the chain of cooperation in order to increase capacity to provide different incentives, and in order to increase the value of the incentive, as well as combining chains of cooperation into networks that make 
the incentive self-sustaining, can help ensure the roles required to execute the process can be reliably engaged, if the capacity to include individuals can be scaled to a large enough group.

One of the features of GCI is functional adaptation (adaptive problem-solving). Applying a collective problem-solving process using GCI, any process and any properties of that process, including choice of individual executing it, might be adapted to maximize any targeted outcome. Applying GCI and it's functional adaptation to the problem of successfully executing a target process, GCI might be used to collaborative execute an adaptive process until the properties of the target process are changed to the point that solving the problem of successfully executing the process is reliably achievable. One example of an adaptive process is increasing the degree of generalization, going from a more specific problem to a more and more general one, until the problem is general enough to be reliably solvable.

Because any GCI based process must build an ever increasing subset of GCI functionality in an cycle that leverages the individuals in one iteration to engage more individuals for the next, it must also have an iterative process of engagement capable of operating within the iterative building cycle. In order to solve the process of engagement for every process, that ever increasing GCI functionality must be used to execute a process of adaptation on that process of engagement.

As an example of one process of engagement, assume a different process is required to engage all $\mathrm{N}$ roles required to perform some process $\mathrm{P}_{\mathrm{B}}$ that builds $\mathrm{GCI}$ functionality in any such cycle. Assume process $P_{1}$ is required to engage role 1 , process $P_{2}$ is required to engage role 2 , and so forth up until process $\mathrm{P}_{\mathrm{N}}$ is required to engage role $\mathrm{N}$.

\section{Generalizing the Process of Engagement}

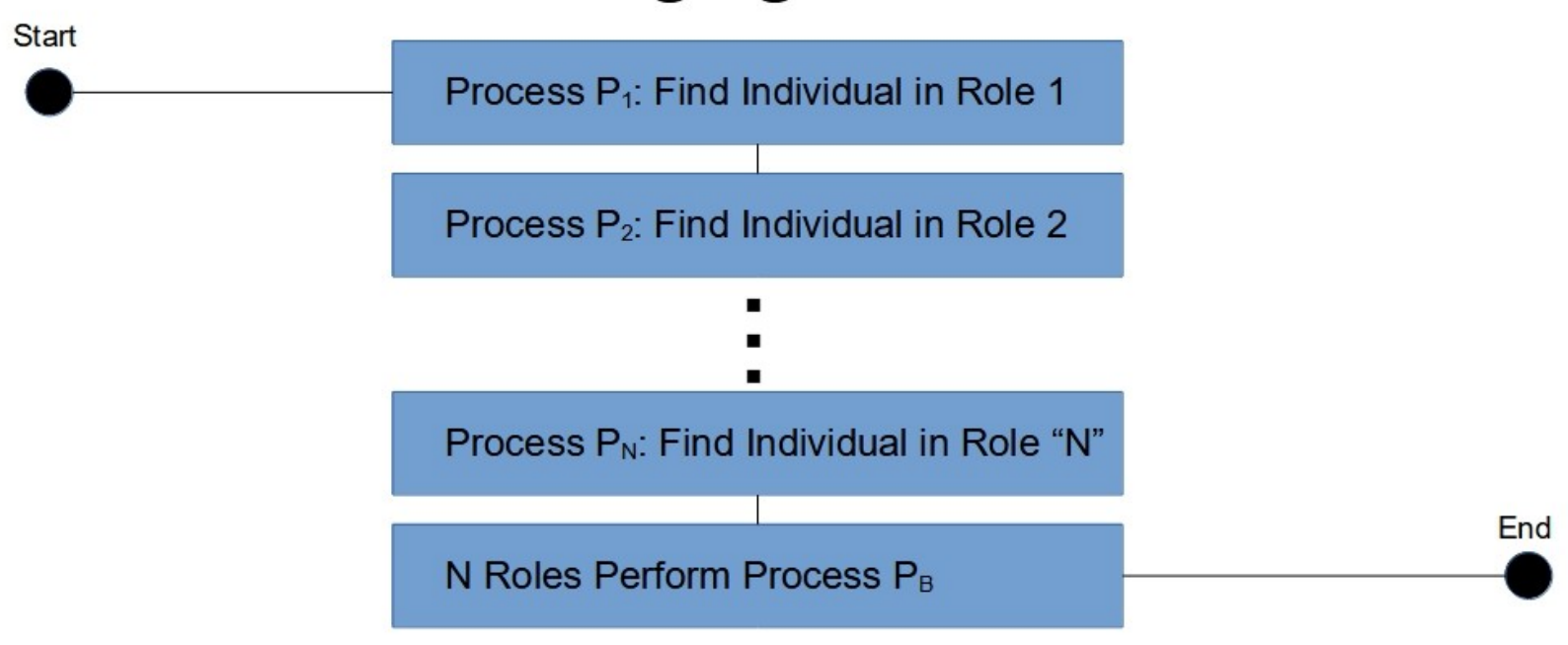

Figure 7: A process for engaging all roles required to build a GCI.

In the case that process $P_{1}$ must be executed to engage role 1 before process $P_{2}$ can be executed to engage role 2 , process $P_{2}$ must be executed to engage role 2 before process $P_{3}$ can be executed to engage role 3 , and so forth until process $\mathrm{P}_{\mathrm{N}-1}$ must be executed to engage role N-1 so that process $\mathrm{P}_{\mathrm{N}}$ 
can be executed to engage role $\mathrm{N}$, and together those $\mathrm{N}$ roles will perform the task $\mathrm{P}_{\mathrm{T}}$, then any one of these steps might be skipped if not required to get to the end result of engaging individuals. That is, if an individual in a role already exists, the process to find that individual doesn't need to be executed. This pattern might be generalized to any task $\mathrm{P}_{\mathrm{T}}$ needing as many as $\mathrm{N}$ roles to reliably engage and incentivize those $\mathrm{N}$ individuals to perform that task.

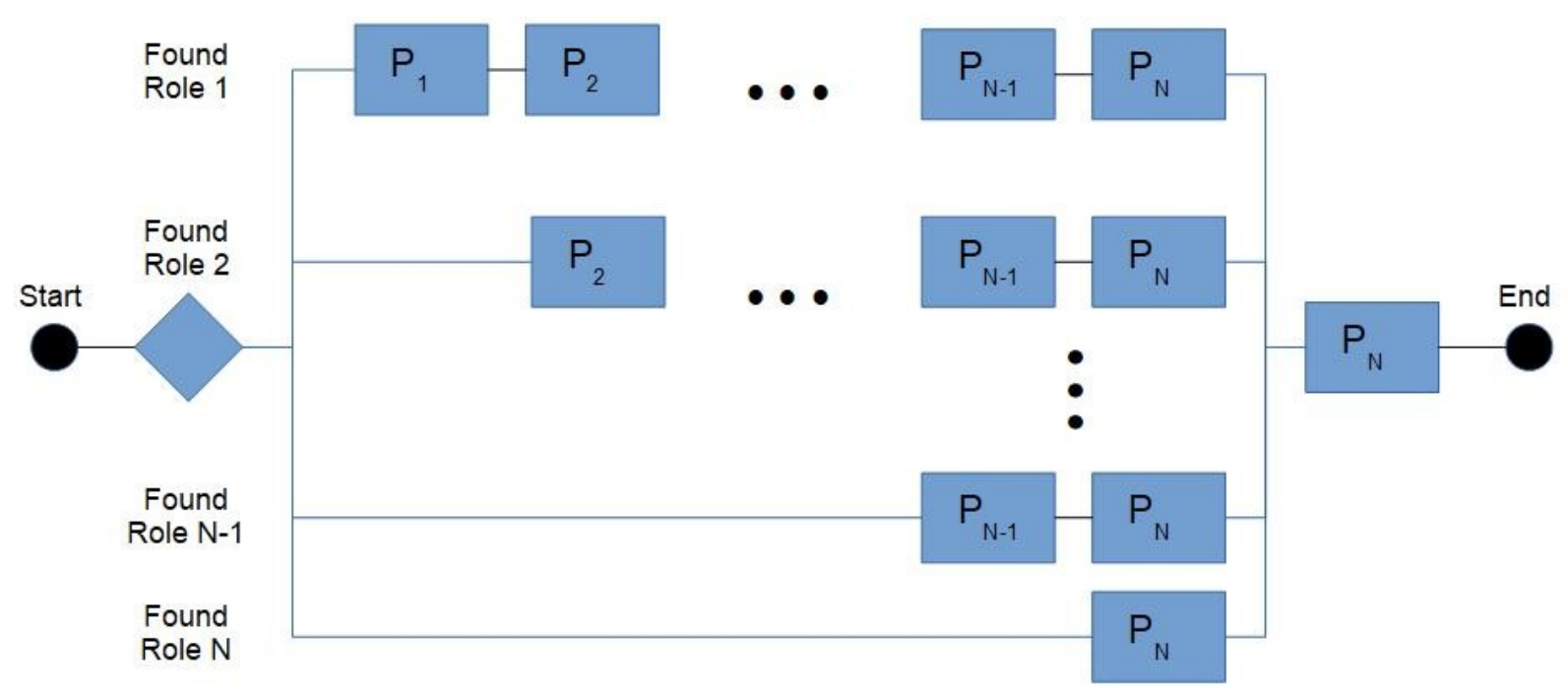

Figure 8: Abstracting the process of engagement might enable that same process to be used elsewhere. Abstracting the processes of engagement in this way helps to ensure such patterns might be reused where they apply. Using GCI to sort through potentially billions of such patterns enables the patterns that are most fit at achieving targeted outcomes in each context to be identified and reused in this way.

\section{Design of an Experiment to Implement a Subset of GCI}

An experiment has been designed to use a subset of GCI to launch a social impact program that will iteratively use a subset of GCI to build an even larger subset of GCI capable of successively increasing that impac. This experiment was designed to make its own execution reliably achieved by splitting the task into small enough blocks that each can reliably be achieved within the skill set available. In this experiment a number of aligned social impact causes are combined into a chain of cooperation in which each participant has the opportunity to have GCI applied to their own social impact cause, to show that individual how it might be used to significantly increase their impact on that cause. In doing so this experiment is intended to demonstrate that GCI can be used to remove barriers of complexity and barriers to scaling cooperation in order to make launch of GCI reliably achievable, as one example of a complex solution.

The experimental control will be an attempt to launch GCI by directly appealing to donors with a proposal to launch GCI, using lack of feedback and lack of follow-up by those donors to show that the level of complexity and hence level of effort required to understand GCI, and the level of cooperation required for donors to self-organize in a way which distributes that effort to the point that it is achieved, all contribute to creating barriers of complexity and barriers to scaling cooperation that make launch not reliably achievable by this example of a non-GCI method.

Though both experiments have been designed, execution awaits a number of steps that take time. One of these steps is building sufficient mind share, since this cooperation requires a critical mass of 
participants. This in turn might require publication of the concepts in both peer-reviewed journal and popular media. Writing and publishing such work of course takes time.

\section{Implications}

Deploying massive networks of cooperation might fundamentally change the way research, development, sales, and all other activities along the business life-cycle are done. For example, in job creation, rather than deploying a single job training program that does not actually create jobs, an entire network of businesses (including job training) that creates sufficient value internally to be selfsustaining might be deployed. Through doing so, a significant increase on targeted outcomes might be achieved. As an example, a conceptual case study of the agricultural livelihoods program proposed for phase I of the CIPAA-SDGs program [3], suggests that an increase of up to 750 times the job creation impact per program dollar might be achievable.

\section{Conclusions}

Through using a methodical approach, GCI has the potential to engage and deploy massive networks of cooperation with the capacity to target virtually any outcome, with the capacity to make achievement of that outcome more probable, with the capacity to increase the magnitude of that outcome, and with the capacity to make that outcome self-sustaining.

\section{References}

[1] Williams, A. E. (2020, December 2). Summary of a Model for General Collective Intelligence. https://doi.org/10.31730/osf.io/kp3x8

[2] Williams, A. E. (n.d.). The Peer to Peer Social Fabric as a Platform for General Collective Intelligence. Retrieved from osf.io/preprints/africarxiv/qbxfr

[3] Williams, A. E. (2020, April 30). The Collective Intelligence based Program to Accelerate Achievement of the Sustainable Development Goals as a Case Study for Collectively Intelligent Program Design. https://doi.org/10.31235/osf.io/r2dxq 